\title{
The real life application of a procalcitonin-based algorithm to reduce antibiotic exposure in hospitalized patients with community acquired pneumonia: a proof of concept
}

\author{
Filippo Pieralli, Vieri Vannucchi, Maria Vittoria Silverii, Ezilda Ricci, Elena Fissi, Antonio Mancini, \\ Francesca Bacci, Carlo Nozzoli
}

Department of Medicine for the Complexity of Care 1, University Hospital of Careggi, Firenze, Italy

\begin{abstract}
Community-acquired pneumonia (CAP) represents a common cause of hospitalization in Internal Medicine wards and a frequent cause of antibiotic prescription. An approach based on procalcitonin (PCT) algorithm, has shown to reduce the antibiotic exposure without affecting outcome, in patients admitted to hospital for pneumonia We conducted a study to evaluate the role of a PCT algorithm in a real-life perspective, to reduce the length of antibiotic treatment (AT) in patients hospitalized with CAP. We performed a prospective case-control study, enrolling patients with CAP admitted to an Internal Medicine ward of Careggi Hospital, between December 2013 and February 2014. We used a validated algorithm of AT guided by PCT in CAP. Discontinuation of AT was strongly indicated when PCT level was normal or reduced by $80 \%$ in patients with PCT values higher than $10 \mathrm{pg} / \mathrm{mL}$ on admission. PCT was drawn on admission, $24 \mathrm{~h}, 4^{\text {th }}$ day and $6^{\text {th }}$ day from admission. The outcomes evaluated were the length of AT, the length of in hospital stay, mortality and serious adverse clinical events. Forty-six patients were consecutively enrolled. On 20 patients, AT was discontinued according to protocol and on 26 patients the AT was continued on clinical judgement. At the baseline, both groups were similar regarding clinical, laboratory, microbiology characteristics, and CURB-65 score. PCT guidance reduced total antibiotic exposure (5.1 \pm 1.5 vs $9.1 \pm 5.8$ days, $\mathrm{P}=0.005)$ without any increase of mortality or serious clinical event. In our study, a PCT algorithm was a useful and safe tool to guide the AT in patients with CAP, reducing the antibiotic exposure.
\end{abstract}

\section{Introduction}

Community-acquired pneumonia (CAP) is the leading infection-related cause of death in developed coun-

Correspondence: Vieri Vannucchi, Department of Medicine for the Complexity of Care 1, University Hospital of Careggi, Largo Brambilla 3, 50134 Firenze, Italy.

Tel./Fax: +39.055.7946411.

E-mail: vieri.vannucchi@gmail.com

Key words: Procalcitonin; community-acquired pneumonia; antibiotic therapy.

Contributions: FP and VV contributed for the study conception, design, interpretation of data and manuscript drafting; MVS, ER, EF contributed to data acquisition; AM, FB and CN contributed in the critical revision.

Conflict of interest: the authors declare no potential conflict of interest.

Received for publication: 26 August 2015.

Revision received: 12 October 2015.

Accepted for publication: 17 October 2015.

This work is licensed under a Creative Commons Attribution NonCommercial 4.0 License (CC BY-NC 4.0).

CCopyright F. Pieralli et al., 2016

Licensee PAGEPress, Italy

Italian Journal of Medicine 2016; 10:213-218

doi:10.4081/itjm.2016.643 tries and one of the most frequent causes of antibiotic prescription in Internal Medicine wards. ${ }^{1}$ An early and appropriate antibiotic therapy has shown to improve the outcome of patients with CAP reducing in-hospital mortality. ${ }^{2}$ However, the length of the antibiotic treatment represents a controversial and debated issue. ${ }^{3}$ Several available guidelines on CAP, agree to interrupt the antibiotic therapy when a clinical stabilization is achieved according to defined clinical and laboratory criteria with a range of treatment duration suggested between 5 and 14 days. ${ }^{4-6}$ An approach based on procalcitonin (PCT) algorithm, has shown to reduce the antibiotic exposure without affecting outcome, in patients admitted to hospital for pneumonia in several randomized controlled studies. ${ }^{3,7}$ However, the usefulness of a PCT algorithm for the management of antibiotic treatment in a perspective of a real-life clinical practice is still unknown. For this reason, we performed a study to assess the usefulness and safety of a PCT-based algorithm to reduce the antibiotic exposure in a real-life clinical setting of patients with CAP admitted to an Internal Medicine Unit.

\section{Materials and Methods}

\section{Population of the study}

We conducted a prospective, case-control, observational study involving patients with CAP admitted 
to the Internal Medicine Unit 1 of Careggi Hospital, between December 2013 and February 2014.

We enrolled all patients with objective evidence of pneumonia defined by the presence of newly discovered abnormal infiltrates on chest radiograph or computed tomography scan and at least two of the following clinical features consistent with pneumonia: fever $\geq 37.8^{\circ} \mathrm{C}$, chest symptoms (dyspnea, productive cough), abnormal chest signs on physical examination (crepitation, bronchial breathing, pleural effusion). Patients were excluded from the study if they met criteria for hospital acquired pneumonia, healthcare-associated pneumonia (i.e., if they were discharged from hospital in the last 90 days prior to the index event, resident in nursing homes, or on dialysis for end stage renal disease), if they were immunocompromised, or if they received antineoplastic treatment within 60 days of hospital admission. Patients diagnosed with atypical bacterial pneumonia (Legionella pneumoniae, Chlamydia pneumoniae, Mycoplasma pneumoniae) were also excluded.

We recorded demographic data, vital signs, comorbid conditions, routine blood tests on admission and during hospitalization. To assess the severity of CAP the CURB-65 score (confusion, urea, respiratory rate, blood pressure, age $\geq 65$ ) was used. The length of stay and duration of antibiotic therapy exposure were recorded for every patient. The choice of antibiotic treatment was made by the attending physician based on the recommendations of Federation of Associations of Hospital Doctors on Internal Medicine (FADOI) guidelines. ${ }^{8}$ The choice to treat the patient according to a PCT-based algorithm or according to usual care, was left at the discretion of the physician tutor of the clinical path of the patient.

A CAP population observed in the same Internal Medicine Unit from March to December 2014, and not included in the algorithm phase, was used as a reference population to compare clinical and demographic characteristics of the study population.

\section{Procalcitonin-based algorithm}

A PCT-based algorithm to guide antibiotic therapy was set in place by a group of physicians of the unit, based on existing algorithm used in a clinical study. ${ }^{1}$ The protocol was modified according to our clinical reality as shown in Figure 1. Physicians were instructed about the algorithm in two seminars and informational meetings before initiation of the study. In order to facilitate the adherence to the algorithm reminders were included in the electronic chart. The inclusion criteria for the PCT-based algorithm were: hospitalization for CAP and a PCT level on admission $\geq 0.25 \mathrm{ng} / \mathrm{mL}$. The PCT was drawn within the first $24 \mathrm{~h}$ from admission to the hospital (regardless to the onset of CAP) and on the $4^{\text {th }}$ and the $6^{\text {th }}$ day since the admission. Discontinuation of antibiotic treatment, after at least 4 days of therapy, was recommended for PCT levels $<0.25 \mathrm{ng} / \mathrm{mL}$ and for a decrease greater than $80 \%$ in the PCT peak value in patients with PCT values $\geq 10 \mathrm{ng} / \mathrm{mL}$ on admission. The overruling of PCT algorithm was possible whenever a clinical evidence of infection persisted despite normal values of PCT.

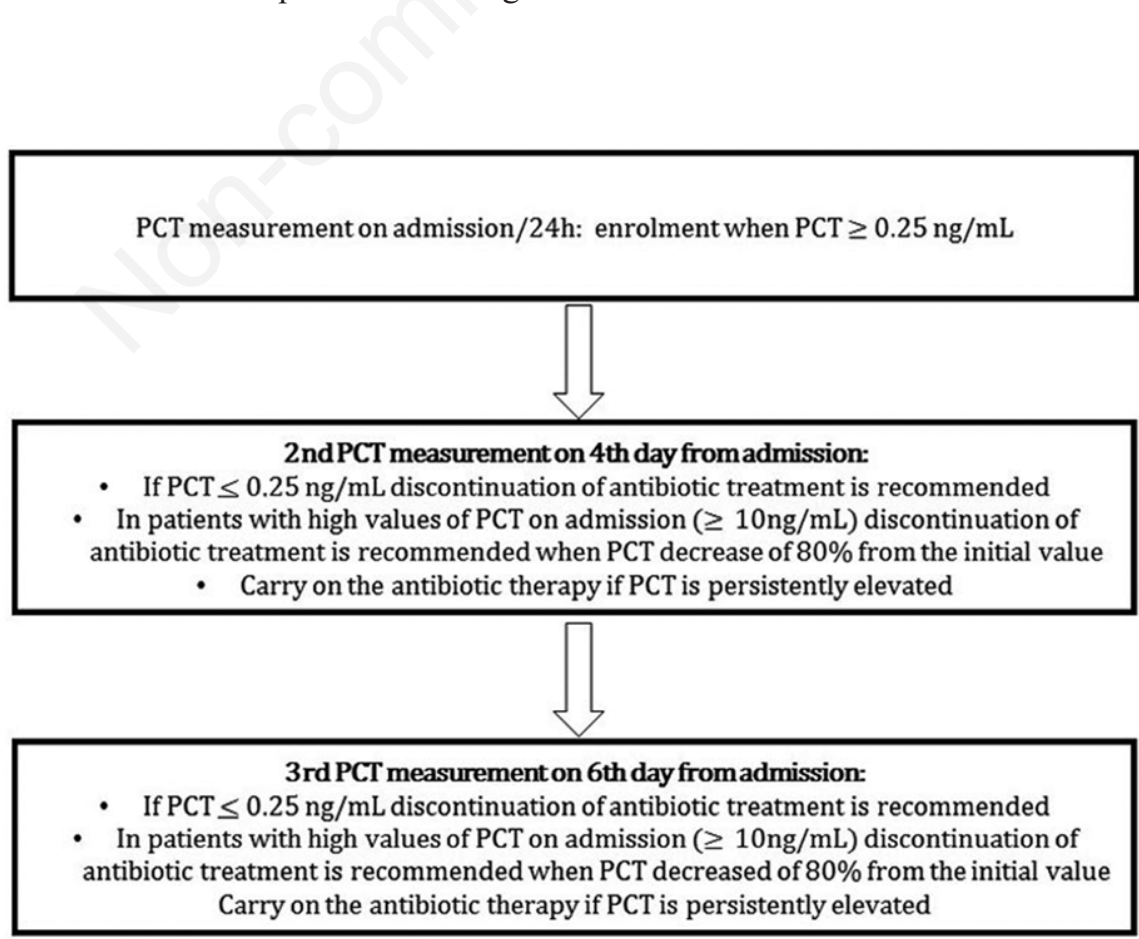

Figure 1. The algorithm based on procalcitonin values to guide the antibiotic therapy in patients with pneumonia. PCT, procalcitonin. 


\section{End-points of the study}

The primary end-points were the evaluation of length of antibiotic exposure and of hospital stay in the PCT-algorithm group with respect to the usual care group. Secondary end-points were: intensive care unit admission, in-hospital mortality, and 30-day mortality and re-hospitalization assessed by standardized phone interviews in those patients discharged alive.

\section{Statistical analysis}

Categorical data were expressed as counts and percentages. Continuous variables were expressed as mean \pm standard deviation. Student's $t$-test or analysis of variance test were used for the comparison of normally distributed continuous data and the Fisher's exact test for the comparison of categorical variables. All P-values were two-tailed and considered significant when $<0.05$ ( $95 \%$ confidence interval). All analyses were performed using the Statistical Package for Social Sciences 19.0 (SPSS Inc., Chicago, IL, USA).

\section{Results}

Forty-six patients were consecutively enrolled from December 2013 to February 2014. The PCT-based antibiotic treatment algorithm was applied in 20 patients, whereas 26 patients were treated with usual care. There was no difference among patients treated according to PCT algorithm and those treated with usual care in terms of demographic, clinical characteristics, comorbidities and severity of disease, assessed by CURB-65 score (Table 1). All patients in both groups were treated according to CAP FADOI guidelines ${ }^{8}$ (Table 1). Of note, mean age was 78 years and both genders were equally represented. Heart failure and chronic obstructive pulmonary disease were the most prevalent comorbidities, and roughly $1 / 3$ of patients had three or more comorbidities. The 46 patients presented demographic characteristics, burden of comorbidity, severity of illness and clinical outcome similar to a population of CAP observed in the same Internal Medicine Unit from March to December 2014.

The PCT values on admission and during hospitalization were significantly higher in patients treated according to PCT algorithm (Table 2). Patients treated according to PCT algorithm presented an important reduction trend of PCT regardless the initial values (Figure 2). The mean length of antibiotic treatment was 7.5 days and was significantly lower in patients treated with the PCT-based algorithm compared to those treated by usual care (5.8 \pm 1.5 days versus $9.1 \pm 5.2 \mathrm{P}=0.005)$, consistent with a reduction of nearly $30 \%$ in antibiotic exposure (Table 3). The length-of-hospital stay was similar in the two groups even if a trend toward a shorter in-hospital length of stay was seen in the PCTbased treatment group (7.3 days versus 9.7 days

Table 1. Demographic and clinical characteristics of the study population.

\begin{tabular}{|c|c|c|c|c|}
\hline \multirow[t]{2}{*}{ Characteristics } & \multirow[t]{2}{*}{ All patients $(n=46)$} & \multicolumn{2}{|c|}{ Adherence to algorithm } & \multirow[t]{2}{*}{$\mathbf{P}$} \\
\hline & & No $(n=26)$ & Yes $(n=20)$ & \\
\hline \multicolumn{5}{|l|}{ Demographics } \\
\hline Age, mean $(y) \pm S D$ & $78.2 \pm 11.6$ & $77.2 \pm 13.8$ & $79.6 \pm 8.1$ & 0.74 \\
\hline Age $\geq 75$ & $32(69.6 \%)$ & $17(65.4 \%)$ & $15(75 \%)$ & 0.53 \\
\hline Male sex, no. $(\%)$ & $25(54.3 \%)$ & $12(46.2 \%)$ & $13(65 \%)$ & 0.24 \\
\hline Smoker & $5(87 \%)$ & $3(11.5 \%)$ & $2(10 \%)$ & 1 \\
\hline Former smoker & $22(47.8 \%)$ & $12(52.2 \%)$ & $10(58.8 \%)$ & 0.75 \\
\hline \multicolumn{5}{|l|}{ Coexisting illnesses, no. (\%) } \\
\hline Renal failure & $8(17.4 \%)$ & $6(23.1 \%)$ & $2(10 \%)$ & 0.43 \\
\hline Diabetes mellitus & $12(26.1 \%)$ & $8(30.8 \%)$ & $4(20 \%)$ & 0.50 \\
\hline Malignancy & $5(10.9 \%)$ & $2(7.7 \%)$ & $3(15 \%)$ & 0.64 \\
\hline Heart failure & $8(17.4 \%)$ & $5(19.2 \%)$ & $3(15 \%)$ & 1 \\
\hline Ischemic heart disease & $21(45.7 \%)$ & $13(50 \%)$ & $12(40 \%)$ & 0.56 \\
\hline Dementia & $9(19.6 \%)$ & $6(23.1 \%)$ & $3(15 \%)$ & 0.71 \\
\hline Liver disease & $2(4.3 \%)$ & $0(0 \%)$ & $2(10 \%)$ & 0.18 \\
\hline Autoimmune disease & $5(10.9 \%)$ & $3(11.5 \%)$ & $2(10 \%)$ & 1 \\
\hline Stroke & $2(4.3 \%)$ & $1(3.8 \%)$ & $1(5 \%)$ & 1 \\
\hline COPD & $13(28.3 \%)$ & $5(19.2 \%)$ & $8(40 \%)$ & 0.18 \\
\hline Coexisting illness $\geq 3$ & $14(30.4 \%)$ & $8(30.8 \%)$ & $6(30 \%)$ & 1 \\
\hline Atrial fibrillation & $12(26.1 \%)$ & $9(34.6 \%)$ & $3(15 \%)$ & 0.18 \\
\hline \multicolumn{5}{|l|}{ CURB 65 score } \\
\hline CURB $65 \geq 3$ & $12(26.1 \%)$ & $6(23.1 \%)$ & $6(30 \%)$ & 0.73 \\
\hline \multicolumn{5}{|l|}{ Antibiotic treatment } \\
\hline According to FADOI guidelines & $46(100 \%)$ & $26(100 \%)$ & $20(100 \%)$ & 1 \\
\hline
\end{tabular}

$\mathrm{SD}$, standard deviation; COPD, chronic obstructive pulmonary disease. 
$\mathrm{P}=0.097)$. No differences in the rate of Intensive Care Unit admission, in-hospital mortality, and 30-day mortality and re-hospitalization were observed between groups (Table 3). All patients, except one, initially assigned to the PCT-based algorithm were treated accordingly, with an adherence to the algorithm of $95 \%$.

\section{Discussion}

In this study we showed that using a PCT-based algorithm significantly reduced the exposition to antibi- otic therapy in patients hospitalized for CAP, without affecting in-hospital and 30-day outcome. Patients treated according to the PCT-based algorithm had a $30 \%$ reduction in antibiotic exposure compared to those treated with usual care. The prolongation of antibiotic treatment in patients with CAP, regardless the clinical improvement and the severity of disease, is a spread and common practice determining several adverse complications related to antibiotics with a consequent increasing of morbidity and costs. ${ }^{9}$ Therefore, in that context the results of this study are of relevance

Table 2. Laboratory findings of the study population.

\begin{tabular}{|c|c|c|c|c|}
\hline \multirow[t]{2}{*}{ Characteristic } & \multirow[t]{2}{*}{ All patients $(n=46)$} & \multicolumn{2}{|c|}{ Adherence to algorithm } & \multirow[t]{2}{*}{$\mathbf{P}$} \\
\hline & & No $(n=26)$ & Yes $(n=20)$ & \\
\hline \multicolumn{5}{|l|}{ Laboratory findings } \\
\hline PCT on admission $(\mathrm{ng} / \mathrm{mL}) \pm \mathrm{SD}^{*}$ & $6.6 \pm 14.3$ & $1.4 \pm 1.5$ & $12.1 \pm 19.2$ & 0.03 \\
\hline PCT $1^{\text {st }}$ day $(\mathrm{ng} / \mathrm{mL}) \pm \mathrm{SD}^{*}$ & $5.7 \pm 8.7$ & $4.3 \pm 6.6$ & $7.3 \pm 10.7$ & 0.29 \\
\hline PCT $4^{\text {th }}$ day $(\mathrm{ng} / \mathrm{mL}) \pm \mathrm{SD}^{*}$ & $2.8 \pm 5.4$ & $2.9 \pm 4.6$ & $2.7 \pm 6.3$ & 0.92 \\
\hline PCT $6^{\text {th }}$ day $(\mathrm{ng} / \mathrm{mL}) \pm \mathrm{SD}^{*}$ & $0.9 \pm 1.5$ & $1 \pm 1.6$ & $0.8 \pm 1.5$ & 0.79 \\
\hline White blood cell count $\left(1000 / \mathrm{mm}^{3}\right) \pm \mathrm{SD}$ & $13.7 \pm 4.8$ & $13.8 \pm 4.3$ & $13.6 \pm 5.5$ & 0.92 \\
\hline Hemoglobin $(\mathrm{g} / \mathrm{dL}) \pm \mathrm{SD}$ & $11.9 \pm 1.3$ & $11.7 \pm 2.3$ & $12.1 \pm 1.9$ & 0.56 \\
\hline Creatinine $(\mathrm{mg} / \mathrm{dL}) \pm \mathrm{SD}$ & $2.9 \pm 10.4$ & $4.1 \pm 13.8$ & $1.3 \pm 0.6$ & 0.32 \\
\hline Urea $(\mathrm{mg} / \mathrm{dL}) \pm \mathrm{SD}$ & $2.2 \pm 9.6$ & $0.8 \pm 0.6$ & $4 \pm 14.6$ & 0.33 \\
\hline Glucose $(\mathrm{g} / \mathrm{L}) \pm \mathrm{SD}$ & $1.4 \pm 0.8$ & $1.4 \pm 0.75$ & $1.5 \pm 0.9$ & 0.66 \\
\hline Sodium $(\mathrm{mEq} / \mathrm{L}) \pm \mathrm{SD}$ & $131.2 \pm 28.4$ & $131.7 \pm 27.2$ & $130.6 \pm 30.7$ & 0.9 \\
\hline $\mathrm{pH} \pm \mathrm{SD}$ & $7.4 \pm 0.1$ & $7.4 \pm 0.1$ & $7.4 \pm 0.1$ & 0.55 \\
\hline $\mathrm{PaO}_{2}(\mathrm{mmHg}) \pm \mathrm{SD}$ & $64.1 \pm 17.6$ & $61.5 \pm 12$ & $66.6 \pm 21.8$ & 0.4 \\
\hline $\mathrm{PaCO}_{2}(\mathrm{mmHg}) \pm \mathrm{SD}$ & $39 \pm 10.2$ & $38.9 \pm 9.9$ & $39.1 \pm 10.8$ & 0.96 \\
\hline
\end{tabular}

PCT, procalcitonin; SD, standard deviation. *Patients treated by usual care lack of some PCT measurements.

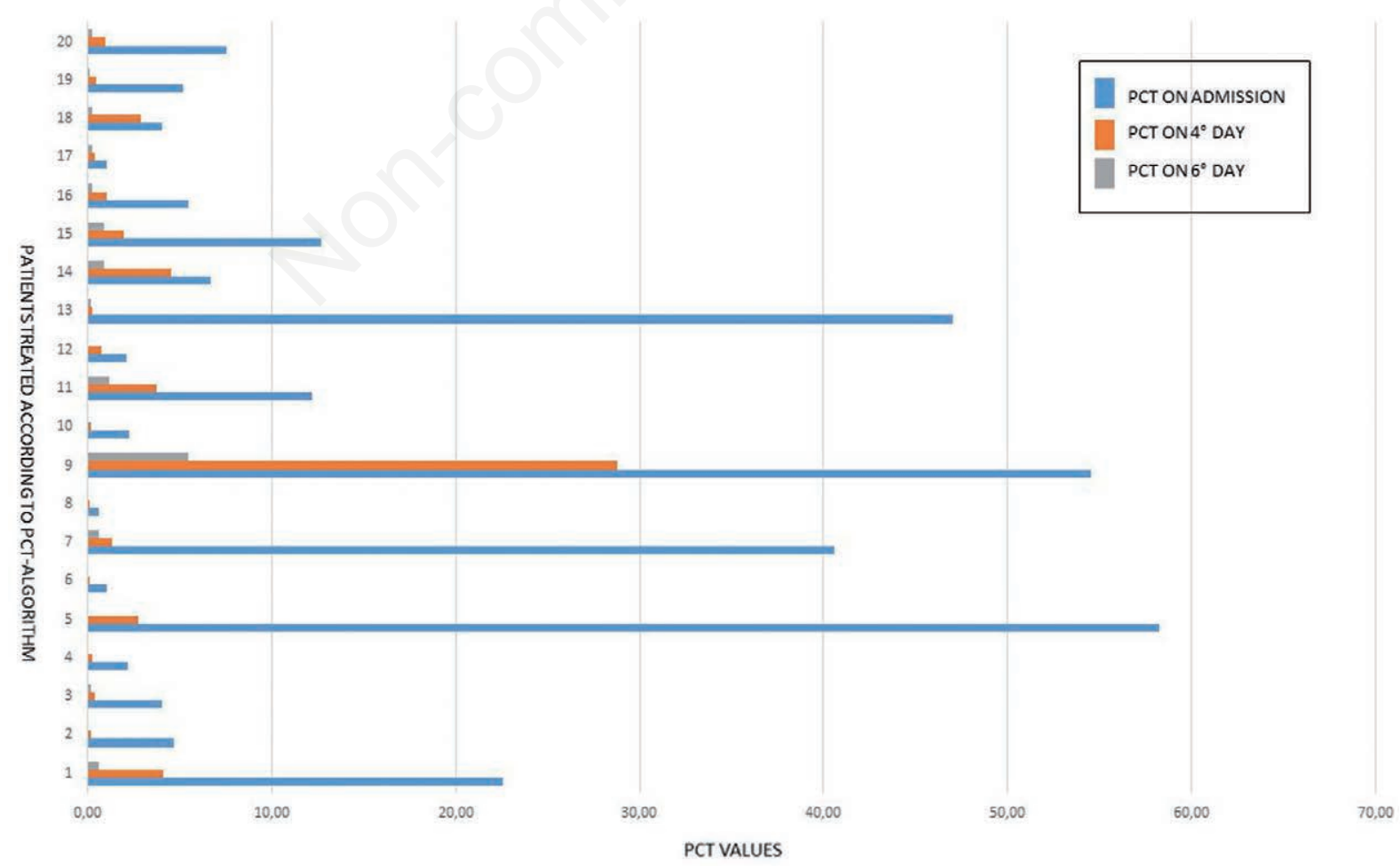

Figure 2. Procalcitonin (PCT) trends of patients treated according to PCT-algorithm. 
since they proved to reduce the duration of antibiotic therapy, so frequently extended in a superfluous manner. Algorithms based on PCT values may be useful tools that integrated with clinical criteria, support physicians on the decision to stop antibiotic therapy. The effectiveness of PCT algorithm to guide antibiotic therapy in patients with CAP was demonstrated in a randomized trial by Christ-Crain and coworkers, where PCT was used as a guidance for the initiation and duration of antibiotic therapy. ${ }^{1}$ The study showed that PCT was a useful guidance to reduce antibiotic prescription on admission and antibiotic treatment duration compared to patients treated with conventional care. A similar study by Schuetz et al. evaluated the effectiveness and safety of procalcitonin-guided antibiotic therapy in lower respiratory tract infections in a real life context. This was an international, multicenter observational quality survey that showed a significant reduction in antibiotic exposure in the PCT group compared to the control group [5.9 vs 7.4 days; difference, -1.51 days; $95 \%$ confidence interval $(\mathrm{CI}),-2.04$ to $-0.98 ; \mathrm{P}<0.001]$, with no risk increase in the combined adverse outcome end point within 30 days of followup when the PCT algorithm was followed regarding early cessation of antibiotics (adjusted odds ratio, 0.61; $95 \%$ CI, 0.36 to $1.04 ; \mathrm{P}=0.07) .{ }^{10}$ However, the population studied was heterogeneous with a prevalence of nearly $30 \%$ of acute bronchitis and acute exacerbations of chronic obstructive pulmonary disease, which are lower respiratory tract infections far less severe than $\mathrm{CAP}$ in hospitalized patients. Furthermore, disease severity assessed by CURB-65 in CAP patients in the study, was less than in a population of real-life CAP patients commonly observed and cared in medical wards (mean/median 1.1/0 vs 1.8/2 in our population).

The reduction in antibiotic exposure represents an important issue especially in elderly patients with several comorbidities where a prolonged antibiotic treatment may induce serious complications. ${ }^{11}$ In our cohort almost $70 \%$ of patients were older than 75 years with a high burden of coexisting comorbidities. Elderly patients frequently have concomitant renal im- pairment that could lead to reduced drug clearance and increased toxicity. ${ }^{11}$ Prolonged antibiotic exposure is the leading risk factor for Clostridium difficile infection, an increasingly recognized health-care associated disease burdened by a high risk of mortality especially in elderly patients. ${ }^{12}$ Moreover, antibiotic pressure is the key factor in promoting antibiotic resistance and the emergence of superinfection by multidrug resistant bacteria which are particularly hazardous in elderly people. For all these reasons, every effort is needed to reduce antibiotic exposure to obtain effective results with the minimal duration of antibiotic therapy. ${ }^{13}$

In our study the use of a PCT-algorithm was not associated with a statistically significant reduction in hospital stay even though patients managed according to the PCT-algorithm presented a trend toward a shorter mean hospital stay than controls (7.3 days versus 9.7 days $\mathrm{P}=0.097)$. This result is likely due to the limited number of patients included in the study, and in general the impact of a PCT-based algorithm on the hospital stay in CAP is not yet known as shown in other studies, where the in-hospital length of stay was similar in the active group and controls. ${ }^{10,13}$ Our results along with those of the above cited studies are appealing, and the way to minimize antibiotic treatment duration without affecting safety, may be an important issue to reduce hospital stay and costs.

This study presents several limitations such as the limited sample size, the single center experience and the limited duration of the period of observation. The usefulness of a PCT-based algorithm was tested over a period of three months and we do not have information about the reliability when using this algorithm for a longer period of time. However, few points of strength should be emphasized. Our cohort represents a typical population of patients daily hospitalized in internal medicine wards for CAP in Italy, and clinical characteristics of the study population are consistent with a larger population of patients hospitalized for $\mathrm{CAP}$ at the same center during one year of observation. One more point of strength of this study is the very low rate of overruling the algorithm. All patients

Table 3. Primary and secondary endpoints of the study.

\begin{tabular}{lcccc}
\hline & All patients & \multicolumn{2}{c}{ Adherence to algorithm } & Y \\
& $(\mathbf{n = 4 6 )}$ & No (n=26) & Yes $(\mathbf{n}=\mathbf{2 0})$ \\
\hline Primary endpoints & & & & \\
$\quad$ Antibiotic duration (days) & $7.65 \pm 4.3$ & $9.1 \pm 5.2$ & $5.8 \pm 1.5$ & $<0.05$ \\
Length of hospital stay (days) & $8.67 \pm 5.1$ & $9.7 \pm 5.8$ & $7.3 \pm 3.5$ & 0.097 \\
\hline Secondary endpoints & & & & \\
ICU admission & $2(4.3 \%)$ & $2(7.7 \%)$ & 0 & 0.5 \\
In-hospital mortality & $1(2.2 \%)$ & $1(3.8 \%)$ & 0 & 0.98 \\
Readmission within 30 days & $4(9.3 \%)$ & $3(12.5 \%)$ & $1(5.3 \%)$ & 0.62 \\
Death within 30 days & $5(10.8 \%)$ & $4(15.3 \%)$ & $1(5.3 \%)$ & 0.62 \\
\hline
\end{tabular}

ICU, Intensive Care Unit. 
but one (95\%) initially treated with the PCT-based algorithm were treated accordingly. The adherence to the algorithm in CAP patients is reported between 35 and $80 \%$ in the real-life context of the proREAL study. ${ }^{10}$ This depends on factors such as the experience with PCT guided management strategies and PCT culture overall. In experienced centers the overall algorithm compliance has been of $90 \%$ outside of study conditions in hospital well used to the algorithm..${ }^{14} \mathrm{At}$ our center, the experience with PCT use was in place long before the study initiation, introductory seminars to PCT use were set in place before the study started and the adherence to the algorithm use was promoted though the electronic chart application. All these can be responsible for the extremely high percentage of algorithm adherence and consequently the minimal rate of overruling.

Finally, one of the most important concerns about the use of PCT is about its cost-benefit ratio. The cost of single PCT measurement is in the range of 10-30 dollars, and it is evident that saving on average two days of antibiotic treatment following a PCT-based algorithm readily determines an advantage on sanitary expenditure. Moreover, PCT may reduce the use of less specific markers of infections such as $\mathrm{C}$-reactive protein or white blood count, with a possible reduction of the global spending. ${ }^{15}$

In conclusion, the use of a PCT-based algorithm significantly reduced the duration of antibiotic therapy without affecting outcome in a typical population of patients hospitalized for CAP in an internal medicine ward. Patients effectively treated according to the PCTbased algorithm had a $30 \%$ reduction in antibiotic exposure compared to those treated with usual care.

\section{References}

1. Christ-Crain M, Stolz D, Bingisser R, et al. Procalcitonin guidance of antibiotic therapy in community-acquired pneumonia: a randomized trial. Am J Respir Crit Care Med 2006;174:84-93.

2. Waterer G, Bennet L. Improving outcomes from community-acquired pneumonia. Curr Opin Pulm Med 2015;21:219-25.

3. File TM Jr, Mandell LA. What is optimal antimicrobial therapy for bacteremic pneumococcal pneumonia? Clin Infect Dis 2003;36:396-8.

4. Mandell LA, Wunderink RG, Anzueto A, et al. Infectious Disease Society of America/American Thoracic Society consensus guidelines on the management of community-acquired pneumonia in adults. CID 2007; 44:s27-72.

5. Lim WS, Baudouin SV, George RC, et al. BTS guidelines for the management of community acquired pneumonia in adults: update 2009. Thorax 2009;64:3.

6. National Institute for Health and Care Excellence (NICE). Pneumonia in adults: diagnosis and management. NICE guidelines [CG191]; Published: December 2014. Available from: https://www.nice.org.uk/guidance/cg191?unlid=2277266692015630214950

7. Bouadma L, Luyt CE, Tubach F, et al.Use of procalcitonin to reduce patients' exposure to antibiotics in intensive care units (PRORATA trial): a multicentre randomised controlled trial. Lancet 2010;375:463-74.

8. Iori I, Gussoni G, Blasi F, et al. Guidelines and management of hospitalized patients with community-acquired pneumonia: the Italian experience of the FASTCAP study. Ital J Med 2008;2:5-18.

9. Aliberti S, Giuliani F, Ramirez J, et al. How to choose the duration of antibiotic therapy in patients with pneumonia. Curr Opin Infect Dis 2015;28:177-84.

10. Schuetz P, Batschwaroff M, Dusemund F, et al. Effectiveness of a procalcitonin algorithm to guide antibiotic therapy in respiratory tract infections outside of study conditions: a post-study survey. Eur J Clin Microbiol Infect Dis 2010;29:269-77.

11. Fein AM. Pneumonia in the elderly: overview of diagnostic and therapeutic approaches. Clin Infect Dis 1999;28:726-9.

12. Jones AM, Kuijper EJ, Wilcox MH. Clostridium difficile: a European perspective. J Infect 2013;66:115-28.

13. Schuetz P, Christ-Crain M, Thomann R, et al. Effect of procalcitonin-based guidelines vs standard guidelines on antibiotic use in lower respiratory tract infections: the ProHOSP randomized controlled trial. JAMA 2009;302: 1059-66.

14. Schuetz P, Batschwaroff M, Dusemund F, et al. Effectiveness of a procalcitonin algorithm to guide antibiotic therapy in respiratory tract infections outside of study conditions: a post-study survey. Eur J Clin Microbiol Infect Dis 2010;29:269-77.

15. Christ-Crain M, Stolz D, Bingisser R, et al. Procalcitonin guidance of antibiotic therapy in community-acquired pneumonia: a randomized trial. Am J Respir Crit Care Med 2006;174:84-93. 